\title{
Women with polycystic ovarian syndrome had excess hair, irregular or absent menstruation, and infertility and felt freakish, abnormal, and not proper women
}

\author{
Kitzinger C, Willmott J. 'The thief of womanhood' : women's experience of polycystic ovarian syndrome. Soc Sci Med \\ 2002;54:349-61.

\section{QUESTIONS: What are the experiences of women with polycystic ovarian syndrome (PCOS)? How do they negotiate their identities as women?}

\author{
Design \\ Exploratory qualitative design.
}

\section{Setting \\ United Kingdom.}

\section{Participants}

30 women (mean age 29 y, 80\% white) with PCOS recruited through a mailing to members of a national PCOS self help organisation.

Source of funding: no external funding.

For correspondence: Dr C Kitzinger,

Department of

Sociology, University of

York, York, UK.

celia_kitzinger@

yahoo.com

\begin{abstract}
Methods
Indepth, semistructured, tape recorded interviews were conducted in the women's homes and lasted 45-90 minutes. The interview schedule was broad based and wide ranging, and was designed to allow women to tell their own stories. The interview schedule had open ended questions with prompts and follow up questions.
\end{abstract}

\section{COMMENTARY}

By exploring the experience of women, this study by Kitzinger and Willmott begins to fill a void in the understanding of PCOS, otherwise dominated by medical and pathologising discourses. As well, the study findings starkly highlight the ways in which dominant images of femininity and womanhood shape health and health care.

Kitzinger and Willmott draw effectively on a wide range of literature to illustrate how cultural standards of feminine beauty and normality shape the experiences of women with PCOS, and by extension, women in general. They also show how feminists have been complicit with such standards by inattention to issues of facial and body hair, and by often equating menstruation and motherhood with womanhood.

As with many qualitative studies, the major limitation of this study lies in the homogeneity of the sample. Despite some ethnic and employment diversity, the participants were primarily white, able bodied, heterosexual women, chosen partly for "geographical ease" in the UK. Thus, it was not possible to explore how different cultural contexts shaped experiences of PCOS and its symptoms. It would be useful to relate the findings to cultural studies of femininity, beauty, and body image and to conduct similar research with a more diverse sample.

The findings have implications for everyday practice in all areas of nursing, although the authors focus on those for women with PCOS. Intriguingly, they show that simplistic (and not necessarily accurate) construction of PCOS as excess "male" hormones may contribute to women's feelings of freakishness. This study underscores why healthcare providers must temper explanations and understanding of health concerns with awareness of how dominant views affect perceptions and choices for themselves and their clients.

Kitzinger and Willmott hope their study will contribute to destigmitising PCOS. It is unclear how we can best help healthcare providers and their clients to resist powerful social images. The study also identifies the need for further research to determine how providers and women might counter rather than comply with practices and discourses that marginalise, stigmatise, and silence.

Colleen Varcoe, RN, PhD Associate Professor, School of Nursing University of Victoria Vancouver, British Columbia, Canada
Data were transcribed using simple orthographic notation, and analysis relied on organising sections of the data into recurrent themes.

\section{Main findings}

PCOS was very important in the everyday lives of most participants. Many women talked about the frustration and anger they felt about delays in diagnosis, the lack of information provided by health professionals, and what they experienced as a general unwillingness of health professionals to take their symptoms seriously.

Thematic analysis revealed pervasive reports of feeling freakish, abnormal, and not proper women. These feelings were related to 3 symptoms commonly experienced by these women and found to be the most distressing: "excess" facial and body hair growth; irregular, absent, or disrupted menstrual periods; and infertility. Smooth hairless bodies and faces, regular menstruation, and the capacity to bear children were associated with femininity. Thus, these women questioned their ability to be defined as "normal women": two thirds said they felt "different" or "abnormal", and 9 women specifically used the term "freaks" to describe their experience of themselves. Women talked repeatedly about wanting to feel like "normal women".

The social spectre of the "bearded lady" loomed in the experience of many of the women. Women experienced their bodies and especially facial hair as a betrayal of their identities as women, and all of them took steps to remove it. Because of their daily private hair removal, many women found themselves restricted in their movements, unable to take unexpected overnight trips or holidays. Women talked about the separateness that occurred as a result of disrupted or absent menstruation. Regular menses were desirable, as a sign of womanhood and fertility. These women experienced an added burden of secrecy because they felt that revealing their irregular, disrupted, or absent menstruation could lead to scrutiny of their identities as women. The fertility implications of PCOS added to the women's feelings of freakishness and of not being real women. Despite the pain caused by infertility, several women said that it was relatively easy to disclose this condition compared with the other symptoms of PCOS.

\section{Conclusions}

Women with polycystic ovarian syndrome felt they were freakish, abnormal, and not proper women. These feelings were associated with excess hair growth; irregular, absent, or disrupted menstruation; and infertility symptoms that distanced them from the dominant feminine ideal. 